\title{
Efficient Testing of Concurrent Programs with Abstraction-Guided Symbolic Execution
}

\author{
Neha Rungta, Eric G Mercer and Willem Visser* \\ Dept. of Computer Science, Brigham Young University, Provo, UT 84602, USA \\ *SEVEN Networks, 901 Marshall Street, Redwood City, CA 94063, USA
}

\begin{abstract}
Exhaustive search techniques such as model checking and symbolic execution are insufficient to detect errors in concurrent programs. In this work we present an abstraction-guided symbolic execution technique that quickly detects errors in concurrent programs that arise from thread schedules and input data values. An abstract system is generated that contains a set of key program locations that are relevant in testing the feasibility of a possible error in the program. We guide a symbolic execution along locations in the abstract system in an effort to generate a corresponding feasible execution trace to the error location. A combination of heuristics are used to automatically rank thread and data non-determinism in order to guide the execution. We demonstrate empirically that abstraction-guided symbolic execution generates feasible execution paths in the actual system to find concurrency errors in a few seconds where exhaustive symbolic execution fails to find the same errors in an hour.
\end{abstract}

\section{Introduction}

The current trend of multi-core and multi-processor computing is causing a paradigm shift from inherently sequential to highly concurrent and parallel applications. Certain thread interleavings, data input values, or combinations of both often cause errors in the system. Systematic verification techniques such as explicit state model checking and symbolic execution are extensively used to detect errors in such systems $[9,25,7,11,16]$.

Explicit state model checking enumerates all possible thread schedules and input data values of a program in order to check for errors $[9,25]$. Whereas symbolic execution techniques substitute certain data values with symbolic values while all other values are concrete $[11,24,16]$. Explicit state model checking and symbolic execution techniques used in conjunction with exhaustive search techniques such as depth-first search are unable to detect errors in medium to large-sized concurrent programs because the number of behaviors caused by data and thread non-determinism is extremely large.

In this work we present an abstraction-guided symbolic execution technique that efficiently detects errors caused by a combination of thread schedules and data values in concurrent programs. The technique automatically identifies a set of key program locations that can potentially lead to an error state in the system. 
The symbolic execution is then guided along these locations in an attempt to generate a feasible execution path to the error state. This allows the execution to focus in parts of the behavior space that are more likely to contain an error and decrease the total time required to detect errors.

A set of target locations that represent a possible error in the program is provided as input to generate an abstract system. The input target locations are either generated from static analysis warnings, imprecise dynamic analysis techniques, or user-specified reachability properties. We automatically generate an abstract system containing locations that are relevant in verifying the reachability of the target locations using control and data dependence analyses. The abstract system contains call sites, conditional branch statements, data definitions, and synchronization points in the program that lie along control paths from the start of the program to the target locations. The program locations in the abstract system do not contain any thread information or data values.

We systematically guide the symbolic execution toward locations in the abstract system in order to reach the target locations. A combination of heuristics are used to automatically pick thread identifiers and input data values at points of thread and data non-determinism respectively. The abstract system simply provides locations of interest in reaching the target locations. We do not verify or search the abstract system like most other abstraction refinement techniques [3, 8]. At points in the execution when we are unable to guide the program execution further along a sequence of locations (e.g. a particular conditional statement) we refine the abstract system by adding program statements that re-define the variables of interest.

We demonstrate in an empirical analysis, on benchmarked multi-threaded Java programs and the JDK 1.4 concurrent libraries, that locations in the abstract system can be used to generate feasible execution paths to the target locations. We show that the abstraction guided-technique can find errors in multithreaded Java programs in a few seconds where exhaustive symbolic execution is unable to find the errors within a time bound of an hour.

\section{Overview}

A high-level overview of the technique is shown in Fig. 1.

Input: The input to the technique is a set of target locations, $L_{t}$, that represent a possible error in the program. The target locations can either be generated using a static analysis tool or a user-specified reachability property. The lockset analysis, for example, reports program locations where lock acquisitions by unique threads may lead to a deadlock [5]. In this case, the target locations are the set of lock acquisition locations generated by the lockset analysis.

Abstract System: An abstraction of the program is generated using standard control and data dependence analyses on control flow graphs. Location $l_{3}$ is a single target location in Fig. 1. The possible execution of location $l_{3}$ is control dependent on the true branch of the conditional statement $l_{2}$. Two definitions of variable $a$ at locations $l_{0}$ and $l_{1}$ reach the conditional statement $l_{2}$; hence, 


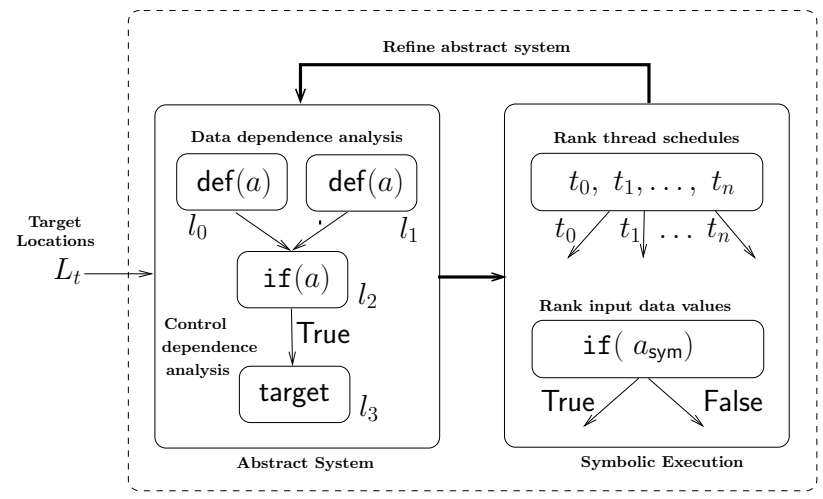

Fig. 1. Overview of the abstraction-guided symbolic execution technique

locations $l_{0}, l_{1}$, and $l_{2}$ are part of the abstract system because they are directly relevant in testing the reachability of $l_{3}$.

Abstraction-Guided Symbolic Execution: The symbolic execution is guided along a sequence of locations (an abstract trace: $\left\langle l_{0}, l_{2}, l_{3}\right\rangle$ ) in the abstract system. The program execution is guided using heuristics to intelligently rank the successor states generated at points of thread and data non-determinism. The guidance strategy uses information that $l_{3}$ is control dependent on the true branch of location $l_{2}$ and in the ranking scheme prefers the successor representing the true branch of the conditional statement.

Refinement: When the symbolic execution cannot reach the desired target of a conditional branch statement in an abstract trace, we refine the abstract system and the abstract trace by adding program statements that re-define the variables in the branch predicate. If we cannot generate the successor state for the true branch of the conditional statement while guiding along $\left\langle l_{0}, l_{2}, l_{3}\right\rangle$ in Fig. 1 then the refinement automatically adds another definition of $a$ to the abstract trace resulting in $\left\langle l_{1}, l_{0}, l_{2}, l_{3}\right\rangle$. It is possible that different threads define the variable $a$ at locations $l_{1}$ and $l_{0}$.

Output: When the guided symbolic execution technique discovers a feasible execution path, the path describes various conditions that lead to the target locations.

\section{Program Model and Semantics}

To simplify the presentation of the guided symbolic execution we describe a simple programming model for multi-threaded and object-oriented systems. The restrictions, however, do not apply to the techniques presented in this work and the empirical analysis is conducted on Java programs. Our programs contain conditional branch statements, procedures, basic data types, complex data types supporting polymorphism, threads, exceptions, assertion statements, and 


\begin{tabular}{|c|c|c|}
\hline $\begin{aligned} \text { 1: } & \text { Thread A }\{ \\
2: & \ldots \\
3: & \text { public void run(Element } \text { elem })\{ \\
4: & \text { lock }(\text { elem }) \\
5: & \text { check }(\text { elem }) \\
6: & \text { unlock }(\text { elem }) \\
7: & \} \\
8: & \text { public void check(Element } \text { elem } \\
9: & \text { if elem.e }>9 \\
10: & \text { Throw Exception } \\
11: & \}\} \quad \text { (a) }\end{aligned}$ & $\begin{array}{ll}\text { 1: } & \text { Thread B }\{ \\
2: & \ldots \\
3: & \\
& \text { public void run(Element elem })\{ \\
4: & \text { int } x \quad / * \text { Input Variable } * / \\
5: & \text { if } x>18 \\
6: & \text { lock }(\text { elem }) \\
7: & \text { elem.reset }() \\
8: & \text { unlock }(\text { elem }) \\
9: & \}\} \quad \text { (b) }\end{array}$ & $\begin{array}{l}\text { 1: } \text { Object Element }\{ \\
2: \quad \text { int } e \\
3: \quad \ldots \\
\text { 4: } \text { public Element }()\{ \\
5: \quad e:=1 \\
6: \quad\} \\
7: \quad \text { public void reset }()\{ \\
8: \quad e:=11 \\
9: \quad\}\} \\
\\
\text { (c) }\end{array}$ \\
\hline
\end{tabular}

Fig. 2. An example of a multi-threaded program with two threads: A and B.

an explicit locking mechanism. The threads are separate entities. The programs contain a finite number of threads with no dynamic thread creation. The threads communicate with each other through shared variables and use explicit locks to perform synchronization operations. The program can also seek input for data values from the environment.

In Fig. 2 we present an example of such a multi-threaded program with two threads A and B that communicate with each other through a shared variable, elem, of type Element. Thread A essentially checks the value elem.e at line 9 in Fig. 2(a) while thread B resets the value of elem.e in Fig. 2(b) at line 7 by invoking the reset function shown in Fig. 2(c). We use the simple example in Fig. 2 through the rest of the paper to demonstrate how the guided symbolic execution technique works.

A multi-threaded program, $\mathcal{M}$, is a tuple $\left\langle\mathcal{C}_{s}, V_{c}, D_{\text {sym }}\right\rangle$ where $\mathcal{C}_{s}$ is a set of threads $\left\{\mathcal{T}_{0}, \mathcal{T}_{1}, \ldots, \mathcal{T}_{u-1}\right\}, V_{c}$ is a finite set of concrete variables, and $D_{\text {sym }}$ is a finite set of all data input variables in the system. Each thread, $\mathcal{T}_{i}$, has an unique identifier $i d$ where $i d \rightarrow\{0,1, \ldots, u-1\}$ and a set of local variables.

A runtime environment implements an interleaving semantics over the threads in the program. The runtime environment operates on a program state $s$ that contains: (1) valuations of the variables in $V_{c},(2)$ for each thread, $\mathcal{T}_{i}$, values of its local variables, runtime stack, and its current program location, (3) the symbolic representations and values of the variables in $D_{\text {sym }}$, and (4) a path constraint, $\phi$, (a set of constraints) over the variables in $D_{\text {sym }}$. The path constraint is represented in some first-order logic formula that can be solved using a theorem prover or a constraint solver. The runtime environment provides a set of functions to access certain information in a program state, $s$ :

- getCurrentLoc(s) returns the current program location of the most recently executed thread in state $s$.

- getLoc $(\mathrm{s}, i)$ returns the current program location of the thread with identifier $i$ in state $s$.

- getEnabledThreads(s) returns a set of identifiers of the threads enabled in s. A thread is enabled if it is not blocked (not waiting to acquire a lock). 
Given a program state, $s$, the runtime environment generates a set of successor states, $\left\{s_{0}, s_{1}, \ldots, s_{n}\right\}$. To generate all successors of a given state, the runtime environment systematically generates the successor states based on the following rules $\forall i \in \operatorname{getEnabledThreads}(s) \wedge l:=\operatorname{getLoc}(s, i)$ :

1. If $l$ is a conditional branch with symbolic primitive data types in the branch predicate, $P$, the runtime environment can generate at most two possible successor states. It can assign values to variables in $D_{\text {sym }}$ to satisfy the path constraint $\phi \wedge P$ for the target of the true branch or satisfy its negation $\phi \wedge \neg P$ for the target of the false branch.

2. If $l$ accesses an uninitialized symbolic complex data structure $o_{\text {sym }}$ of type $T$, then the runtime environment generates multiple possible successor states where $o_{\text {sym }}$ is initialized to: (a) null, (b) references to new objects of type $T$ and all its subtypes, and (c) existing references to objects of type $T$ and all its subtypes.

3. If neither rule 1 or 2 are satisfied, then the runtime environment generates a single successor state obtained by executing $l$ in thread $\mathcal{T}_{i}$.

In the initial program state, $s_{0}$, the current program location of each thread is initialized to its corresponding start location while the variables in $D_{\text {sym }}$ are assigned a symbolic value $v_{\perp}$ that represents an uninitialized value.

A state $s_{n}$ is reachable from the initial state $s_{0}$ if using the runtime environment we can find a non-zero sequence of states $\left\langle s_{0}, s_{1}, \ldots, s_{n}\right\rangle$ that leads from $s_{0}$ to $s_{n}$ such that $\forall\left\langle s_{i}, s_{i+1}\right\rangle, s_{i+1}$ is a successor of $s_{i}$ for $0 \leq i \leq n-1$. Such a sequence of program states represents a feasible execution path through the system. The sequence of program states provides a set of concrete data values and a valid path constraint over the symbolic values. The reachable state space, $S$, can be generated using the runtime environment where $S:=\left\{s \mid \exists\left\langle s_{0}, \ldots, s\right\rangle\right\}$.

\section{Abstraction}

In this work we create an abstract system that contains program locations relevant in checking the reachability of the target program locations and use the abstraction to guide the symbolic execution. The control flow of the system and dependence relationships between the program locations and the target locations are used to construct the abstract system.

\subsection{Background definitions}

A control flow graph (CFG) of a procedure in a system is a directed graph $\langle L, E\rangle$ where $L$ is a set of uniquely labeled program locations in the procedure while $E \subseteq L \times L$ is the set of edges that represents the possible flow of execution between the program locations. Each CFG has a start location $l_{\text {start }} \in L$ and an end location $l_{\text {end }} \in L$.

For a system with $p$ procedures the control flow of the entire system is $\langle\mathcal{L}, \mathcal{E}\rangle$ where $\mathcal{L}:=\bigcup_{0 \leq i \leq p} L_{i}$ and $\mathcal{E}:=\bigcup_{0 \leq i \leq p} E_{i}$. We define the following functions to access information about the locations and edges in the CFGs: 
- start $(l)$ returns true iff $l$ is a start location.

- end $(l)$ returns true iff $l$ is an end location.

- callSite $(l)$ returns true iff $l$ is a call site that invokes a procedure.

- $\operatorname{branch}(l)$ returns true iff $l$ is a conditional branch statement.

- aquireLock $(l)$ returns true iff $l$ acquires a lock.

- releaseLock $\left(l, l^{\prime}\right)$ returns true iff $l$ releases a lock that is acquired at $l^{\prime}$.

- callEdge $\left(l, l^{\prime}\right)$ returns true iff callSite $(l) \wedge \operatorname{start}\left(l^{\prime}\right) \wedge l$ invokes $l^{\prime}$.

There are two kinds of paths that denote the control flow in the system: a path within a CFG (intraprocedural path) and a path across different CFGs (interprocedural path). An intraprocedural or interprocedural path is simply a non-zero sequence of locations $\left(q:=\left\langle l_{i} \ldots, l_{i+n}\right\rangle\right)$ that satisfies certain conditions. The intraprocedural and interprocedural paths are defined using the following functions respectively:

- $\operatorname{cfgPath}\left(l_{i}, l_{n}\right)$ returns true iff $\exists\left\langle l_{i}, \ldots, l_{n}\right\rangle,\left(l_{j}, l_{j+1}\right) \in \mathcal{E}$ for $i \leq j \leq n-1$.

- icfgPath $\left(l_{i}, l_{n}\right)$ returns true iff $\exists\left\langle l_{i}, \ldots, l_{n}\right\rangle,\left(l_{j}, l_{j+1}\right) \in \mathcal{E} \vee \operatorname{callEdge}\left(l_{j}, l_{j+1}\right)$ for $i \leq j \leq n-1$.

The icfgPath function describes a path between two locations across different procedures that does not contain any return $\left(l_{\text {end }}\right)$ locations; it is a sequence of calls required to reach a particular procedure.

Data and control dependences are an integral part in constructing the abstract system. The dependence analyses are defined along intraprocedural paths in the CFG. Data dependence is primarily based on reaching definitions; whereas control dependence is determined by whether the outcomes of branch predicates in conditional statements affect the reachability of certain locations. We define the following functions for the dependence analyses as:

- postDom $\left(l_{i}, l_{n}\right)$ returns true iff $\forall\left\langle l_{i}, \ldots, l_{m}\right\rangle, \operatorname{cfgPath}\left(l_{i}, l_{m}\right) \wedge \operatorname{end}\left(l_{m}\right) \wedge \exists\left(l_{j}==\right.$ $\left.l_{n}\right)$ for $i+1 \leq j \leq m$.

- defines $(l, v)$ returns true iff $l$ defines variable $v$.

- uses $(l, v)$ returns true iff $l$ uses variable $v$.

- reaches $\left(l_{i}, l_{n}\right)$ returns true iff $\exists\left\langle l_{i}, \ldots, l_{n}\right\rangle, \operatorname{cfgPath}\left(l_{i}, l_{n}\right) \wedge \operatorname{defines}\left(l_{i}, v\right) \wedge$ $\neg$ defines $\left(l_{j}, v\right) \wedge$ uses $\left(l_{n}, v\right)$ for $i+1 \leq j \leq n-1$.

- controlD $\left(l_{i}, l_{n}\right)$ returns true iff $\exists\left\langle l_{i}, \ldots, l_{n}\right\rangle, \operatorname{cfgPath}\left(l_{i}, l_{n}\right) \wedge \operatorname{branch}\left(l_{i}\right) \wedge$ postDom $\left(l_{j}, l_{n}\right) \wedge \neg$ postDom $\left(l_{i}, l_{n}\right)$ for $i+1 \leq j \leq n-1$.

\subsection{Abstract System}

The locations in an abstract system, $\mathcal{A}$, are a subset of the locations in the control flow of the entire system. The abstract system contains locations that are call sites, conditional branch statements, data definitions, and synchronization operations that lie along control paths from the start of the program to the target locations.

The abstract system is a directed graph $\mathcal{A}:=\left\langle L_{\alpha}, E_{\alpha}\right\rangle$ where $L_{\alpha} \subseteq \mathcal{L}$ is the set of program locations while $E_{\alpha} \subseteq L_{\alpha} \times L_{\alpha}$ is the set of edges. The abstract 
system is constructed based on the set of input target locations $L_{t}$ and the CFGs of the system $\langle\mathcal{L}, \mathcal{E}\rangle$. We initialize the set of abstract locations, $L_{\alpha}$, with the set of target locations $L_{t}$ and the set of all possible start locations of the program $L_{s}$. The set $L_{s}$ contains the start location of each thread, $\mathcal{T}_{i}$, in the system. We initialize the set $L_{\alpha}:=L_{t} \cup L_{s}$ and iteratively add locations, $l \in \mathcal{L}$, to $L_{\alpha}$ if one of the following four equations is satisfied. We continue to add locations until we reach a fixpoint, re-evaluating the four equations each time a location is added.

$$
\begin{array}{r}
\exists l_{t} \in L_{t}, l_{s} \in L_{s}, l^{\prime} \in \mathcal{L},\left[\operatorname{icfgPath}\left(l, l_{t}\right) \wedge \operatorname{icfgPath}\left(l^{\prime}, l_{t}\right)\right] \wedge \\
{\left[\operatorname{icfgPath}\left(l_{s}, l\right) \wedge \operatorname{icfgPath}\left(l_{s}, l^{\prime}\right)\right] \wedge\left[\operatorname{callEdge}\left(l, l^{\prime}\right) \vee \operatorname{callEdge}\left(l^{\prime}, l\right)\right]}
\end{array}
$$

The call sites are added to $L_{\alpha}$ one at a time by satisfying Eq. (1); the call sites are part of method sequences such that invoking a particular sequence leads from the start of the program to a procedure containing a target location. In addition to the call sites, start locations of the procedures invoked by the call sites are also added one at a time to the set of locations $L_{\alpha}$ when Eq. (1) evaluates to true.

$$
\begin{aligned}
& \exists l_{\alpha} \in L_{\alpha}, l^{\prime} \in \mathcal{L},\left[\operatorname{cfgPath}\left(l, l_{\alpha}\right) \wedge \operatorname{cfgPath}\left(l^{\prime}, l_{\alpha}\right)\right] \wedge \\
& \left\{\left[\operatorname{cfgEdge}\left(l, l^{\prime}\right) \wedge \operatorname{controlD}\left(l, l^{\prime}\right) \wedge \operatorname{controlD}\left(l, l_{\alpha}\right)\right] \vee\right. \\
& \left.\left[\operatorname{cfgEdge}\left(l^{\prime}, l\right) \wedge \operatorname{controlD}\left(l^{\prime}, l\right) \wedge \operatorname{controlD}\left(l^{\prime}, l_{\alpha}\right)\right]\right\}
\end{aligned}
$$

Conditional branch statements that determine the reachability of the locations that are already present in the abstract system are added to $L_{\alpha}$ whenever Eq. (2) is satisfied. The branch statements that result from any nested control dependence are also added. Furthermore, the immediate target of a conditional branch statement is added when Eq. (2) evaluates to true where the execution of the target depends on the same branch outcome as $l_{\alpha}$. This allows the desired target of the branch to be encoded in the abstract trace.

$$
\begin{array}{r}
\exists l_{\alpha} \in L_{\alpha}, \operatorname{cfgPath}\left(l, l_{\alpha}\right) \wedge \operatorname{defines}(l, v) \wedge \\
\operatorname{isBranch}\left(l_{\alpha}\right) \wedge \operatorname{uses}\left(l_{\alpha}, v\right) \wedge \operatorname{reaches}\left(l, l_{\alpha}\right)
\end{array}
$$

Locations that define variables used in branch predicates at the conditional statements in $L_{\alpha}$ are added if Eq. (3) is satisfied. To compute the reaching definitions we conservatively compute the alias information based on the notion of maybe an alias. If two variables in a given procedure can be aliases of one another we assume they are aliases. In the reaches function the alias information is only propagated along a path in the CFG; in other words at an intraprocedural level (single procedure). The alias computation does not consider aliases among variables passed as parameters to different procedures. This restriction allows us to generate a smaller set of locations in the abstract system and we rely on refinement to find other definitions if/when they are needed.

$$
\begin{aligned}
\exists l_{\alpha} \in L_{\alpha}, & {\left[\operatorname{cfgPath}\left(l, l_{\alpha}\right) \wedge \text { acquireLock }(l)\right] \vee } \\
& {\left[\operatorname{cfgPath}\left(l_{\alpha}, l\right) \wedge \text { releaseLock }\left(l, l_{\alpha}\right)\right] }
\end{aligned}
$$




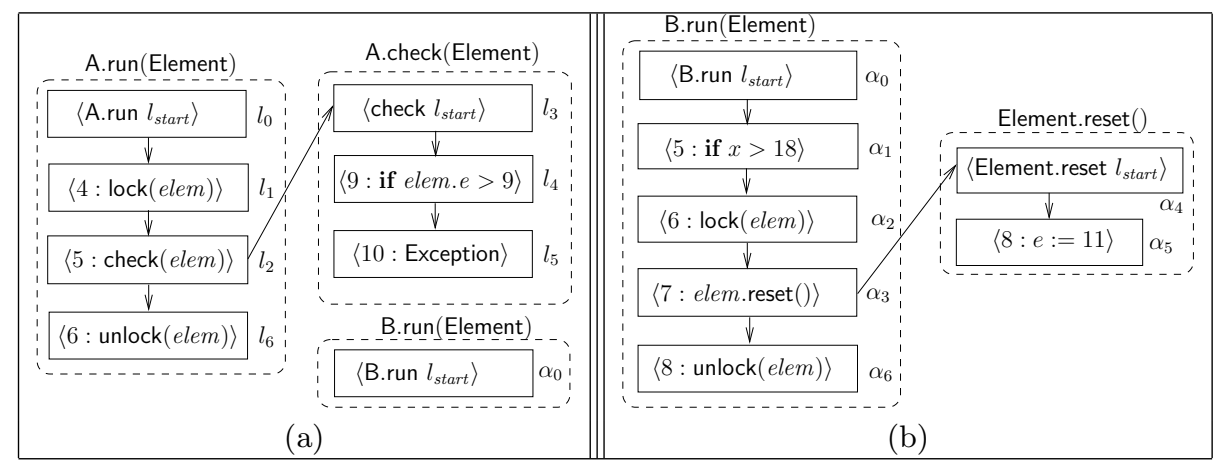

Fig. 3. The abstract system for Fig. 2: (a) Initial Abstract System. (b) Additions to the abstract system after refinement.

Eq. (4) adds locations for synchronization operations. Locations where locks are relinquished corresponding to the lock acquisition locations are also added to $L_{\alpha}$ using the releaseLock function.

Termination: Loops and recursive procedure calls can cause cyclic dependencies between different branch statements, data definitions, and call sites. Program locations that are part of cyclic dependencies, however, are added only once to $L_{\alpha}$ since $L_{\alpha}$ is a set of unique locations; this guarantees termination.

Finally, edges are added between the different locations in the abstract system. An edge between any two locations $l_{\alpha}$ and $l_{\alpha}^{\prime}$ in $L_{\alpha}$ is added to $E_{\alpha}$ if either Eq. (5) or Eq. (6) evaluates to true.

$$
\begin{array}{r}
\exists\left\langle l_{\alpha}, l_{i}, \ldots, l_{n}, l_{\alpha}^{\prime}\right\rangle, \operatorname{cfgPath}\left(l_{\alpha}, l_{\alpha}^{\prime}\right) \wedge l_{j} \notin L_{\alpha} \text { for } i \leq j \leq n \\
\operatorname{callEdge}\left(l_{\alpha}, l_{\alpha}^{\prime}\right)
\end{array}
$$

The abstract system for the example in Fig. 2 where the target location is line 10 in the check method in Fig. 2(a) is shown in Fig. 3(a). Locations $l_{0}$ and $\alpha_{0}$ in Fig. 3(a) are the two start locations of the program. The target location, $l_{5}$, represents line 10 in Fig. 2(a). Location $l_{2}$ is a call site that invokes start location $l_{3}$ that reaches target location $l_{5}$. The target location is control dependent on the conditional statement at line 9 in Fig. 2(a); hence, $l_{4}$ is part of the abstract system in Fig. 3(a). The locations $l_{1}$ and $l_{6}$ are the lock and unlock operations. The abstract system shows Thread B is not currently relevant in testing the reachability of location $l_{5}$.

\subsection{Abstract Trace Set}

The input to the guided symbolic execution is an abstract trace set. The abstract trace set contains sequences of locations generated on the abstract system, $\mathcal{A}$, from the start of the program to the various target locations in $L_{t}$. We refer to 
the sequences generated on the abstract system as abstract traces to distinguish them from the sequences generated on the CFGs. To construct the abstract trace set we first generate intermediate abstract trace sets, $\left\{P_{0}, P_{1}, \ldots P_{t-1}\right\}$, that contain abstract traces between start locations of the program $\left(L_{s}\right)$ and the input target locations $\left(L_{t}\right)$. For each target location, $l_{i} \in L_{t}$, we use Eq. (7) to create a set of abstract traces, $P_{i}$, where each abstract trace leads from the start of the program to $l_{i}$.

$$
\begin{array}{r}
\forall l_{0} \in L_{s}, P_{i}:=\left\{\left\langle l_{0}, l_{1}, \ldots, l_{i}\right\rangle \mid l_{j} \neq l_{k},\left\langle l_{l}, l_{l+1}\right\rangle \in E_{\alpha}\right. \\
\text { for } j \neq k \text { and } 0 \leq j, k \leq i \text { and } 0 \leq l \leq i-1\}
\end{array}
$$

Eq. (7) generates traces of finite length in the presence of cycles in the abstract system caused by loops, recursion, or cyclic dependencies in the program. Eq. (7) ensures that each abstract trace generated does not contain any duplicate locations by not considering any back edges arising from cycles in the abstract system. We rely on the guidance strategy to drive the program execution through the cyclic dependencies toward the next interesting location in the abstract trace; hence, the cyclic dependencies are not encoded in the abstract traces that are generated from the abstract system.

Each intermediate abstract trace set, $P_{i}$, contains several abstract traces from the start of the program to a single target location $l_{i} \in L_{t}$. We generate a set of final abstract trace sets:

$$
\Pi_{\mathcal{A}}:=\left\{\left\{\pi_{0}, \ldots, \pi_{t-1}\right\} \mid \pi_{0} \in P_{0}, \ldots, \pi_{t-1} \in P_{t-1}\right\}
$$

Each $\Pi_{\alpha} \in \Pi_{\mathcal{A}}$ contains a single abstract trace from the start of the program to a target location. In essence, $\Pi_{\alpha}:=\left\{\pi_{\alpha_{0}}, \pi_{\alpha_{1}}, \pi_{\alpha_{t-1}}\right\}$ where each $\pi_{\alpha_{i}} \in \Pi_{\alpha}$ is an abstract trace from the start of the program to a unique $l_{i} \in L_{t}$ and $\left|\Pi_{\alpha}\right|=\left|L_{t}\right|$.

The input to the guided symbolic execution technique is $\Pi_{\alpha} \in \Pi_{\mathcal{A}}$. The different abstract trace sets in $\Pi_{\mathcal{A}}$ allow us to easily distribute checking the feasibility of individual abstract trace sets on a large number of computation nodes. Each execution is completely independent of another and as soon as we find a feasible execution path to the target locations we can simply terminate the other trials.

In the abstract system shown in Fig. 3(a) there is only a single target location-line 10 in check procedure shown in Fig. 2(a). Furthermore, the abstract system only contains one abstract trace leading from the start of the program to the target location. The abstract trace $\Pi_{\alpha}$ is a singleton set containing $\left\langle l_{0}, l_{1}, l_{2}, l_{3}, l_{4}, l_{5}\right\rangle$.

\section{Guided Symbolic Execution}

We guide a symbolic program execution along an abstract trace set, $\Pi_{\alpha}:=$ $\left\{\pi_{0}, \pi_{1}, \ldots, \pi_{t-1}\right\}$, to construct a corresponding feasible execution path, $\Pi_{s}:=$ $\left\langle s_{0}, s_{1}, \ldots, s_{n}\right\rangle$. For an abstract trace set, the guided symbolic execution tries 


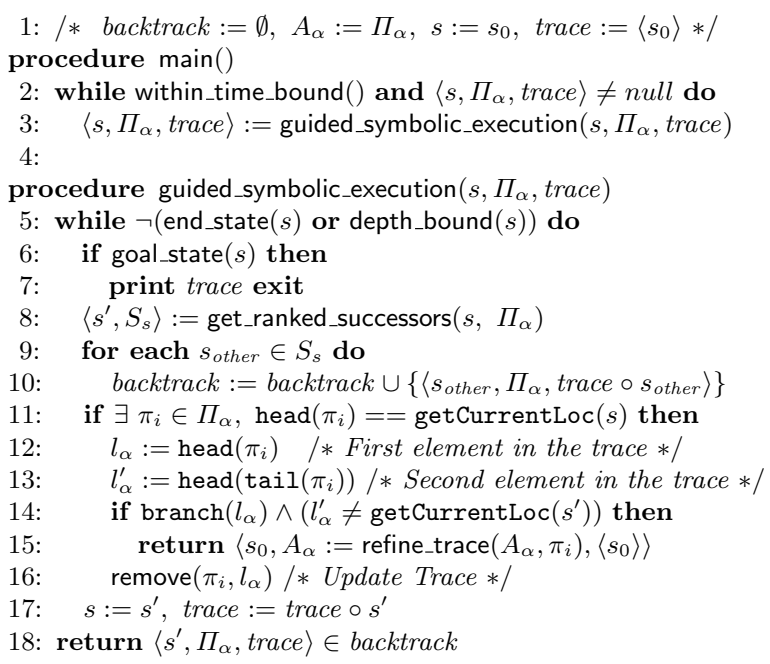

Fig. 4. Guided symbolic execution pseudocode.

to generate a feasible execution path that contains program states where the program location of the most recently executed thread in the state matches a location in the abstract trace. The total number of locations in the abstract trace is $m:=\sum_{\pi_{i} \in \Pi_{\alpha}}$ length $\left(\pi_{i}\right)$ where the length function returns the number of locations in the abstract trace $\pi_{i}$. In our experience, the value of $m$ is a lot smaller than $n, m<<n$ where $n$ is the length of the feasible execution trace corresponding to $\Pi_{\alpha}$ because the abstract traces contain large control flow gaps between two locations in the abstract trace. Intermediate program locations are not included in the abstract system or the resulting abstract traces.

The pseudocode for the guided symbolic execution is presented in Fig. 4. On line 1 we initialize the backtrack set as empty, store a copy of the input abstract trace set $\Pi_{\alpha}$ in $A_{\alpha}$, set program state $s$ to the initial program state $s_{0}$, and add $s_{0}$ to the feasible execution trace. On line 3 , main invokes guided_symbolic_execution where the values of the elements in the tuple are $\left\langle s_{0}, \Pi_{\alpha},\left\langle s_{0}\right\rangle\right\rangle$. A time and depth bound are specified by the user as the termination criteria of the symbolic execution.

The guided symbolic program execution is a greedy depth-first search that picks the best immediate successor of the current state and does not consider unexplored successors until it reaches the end of a path and needs to backtrack. The search is executed along a path in the program until it reaches an end state (a state with no successors), a user-specified depth bound (line 5 ), a user-specified time bound (line 2), or the goal state (line 6). In the goal state, $s$, there exists a unique thread at each target location $\left(\forall l_{i} \in L_{t}, \exists j \in\right.$ getEnabledThreads $\left.(s), \operatorname{getLoc}(s, j)==l_{i}\right)$. If the state $s$ is the goal state (line $6)$ then the feasible execution trace is printed before exiting the search. In this 
scenario we are successfully able to find a corresponding execution trace that includes each location in the abstract trace set. The guided symbolic execution technique is guaranteed to terminate even if the goal state is not reachable because it is depth and time bounded.

States are assigned a heuristic rank in order to intelligently guide the program execution. The get_ranked_successors function returns a tuple $\left\langle s^{\prime}, S_{s}\right\rangle$ on line 8 in Fig. 4 where $s^{\prime}$ is the best ranked successor of state $s$ while all the other successors are in set $S_{s}$. Each $s_{\text {other }} \in S_{s}$ is added to the backtrack set with the abstract trace set and the feasible execution trace (lines 9 and 10). The feasible execution trace added to the backtrack set with $s_{\text {other }}$ denotes a feasible execution path from $s_{0}$ to $s_{\text {other }}$. The best-ranked state $s^{\prime}$ is assigned as the current state and the feasible execution trace is updated by concatenating $s^{\prime}$ to it using the $\circ$ function (line 17).

In order to match a location in the abstract trace set to a program state, the algorithm checks whether the program location of the most recently executed thread in state $s$ matches the first location in an abstract trace, $\pi_{i} \in \Pi_{\alpha}$ (line 11). The head function returns the first element of the input abstract trace. The tail function returns the input abstract trace without its head. Location $l_{\alpha}$ is the first location in $\pi_{i}$ while $l_{\alpha}^{\prime}$ is the immediate successor of $l_{\alpha}$. Location $l_{\alpha}$ is removed from the abstract trace (line 16) if refinement is not needed. Removing $l_{\alpha}$ updates $\pi_{i}$ and in turn updates $\Pi_{\alpha}$. The execution now attempts to match the location of the most recently executed thread in the current state toward the next location in $\pi_{i}$ by directing the search.

The abstract trace set is immediately refined when the program execution is unable to execute the program along the desired target of a conditional branch statement in an abstract trace $\pi_{i}$ on line 15 in Fig. 4 . The refinement is invoked when the current values of the variables in the branch predicate $l_{\alpha}$ do not result in the desired successor $s^{\prime}$ of $s$. The refinement is performed on the abstract trace set $A_{\alpha}$ (a copy of the original unmodified abstract trace set $\Pi_{\alpha}$ ). After the refinement the search is restarted from the initial program state $s_{0}$ and the updated abstract trace set $A_{\alpha}$. The details on the refinement process are given in Section 6.

The get_ranked_successors $\left(s, \Pi_{\alpha}\right)$ in Fig. 4 takes as input a program state $s$ and the abstract trace set $\Pi_{\alpha}$. The runtime environment generates a set of successors $\left\{s_{0}^{\prime}, s_{1}^{\prime}, \ldots, s_{x}^{\prime}\right\}$ for the program state $s$. For each successor state $s_{i}^{\prime}$, we compute its heuristic value using a two-tier and data ranking scheme. The two-tier ranking scheme has been described in earlier works $[18,19]$ while ranking the data non-determinism is new to this work.

Two-tier Rank: $h_{1}\left(s_{i}^{\prime}\right):=\sum_{\pi_{j} \in \Pi_{\alpha}}$ length $\left(\pi_{j}\right)$ is the first-level rank of $s$. Intuitively, program states along execution paths that correspond to more locations from the input abstract trace set are ranked better than others [18]. States with lower heuristic values are ranked higher. The second-level rank is used to prioritize the states that have the same first-level rank. The secondlevel rank of $s_{i}^{\prime}$ is an estimate of the distance from the program state to the 


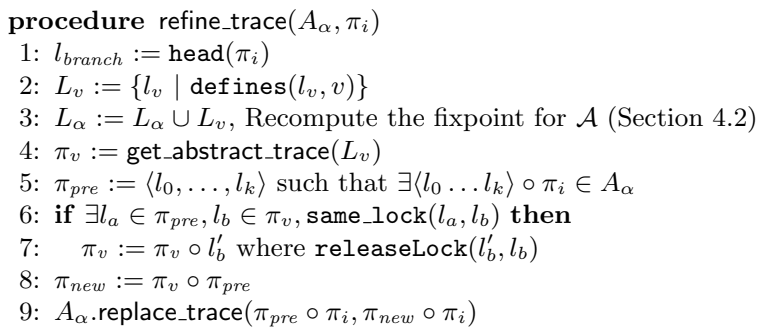

Fig. 5. Refinement pseudocode.

next program location in any of the abstract traces in $\Pi_{\alpha}$ [19]. A high-level description of the second-level rank is provided in a technical report [20].

Ranking Data Non-Determinism: New in this work we rank the nondeterministic choices that are generated for complex input data structures. We rank $s_{i}^{\prime}$ at a point of complex data non-determinism for some object $o b j_{s y m}$. If there exists in an abstract trace in $\Pi_{\alpha}$ a call site $l$ where $o b j_{\text {sym }}$ is the object that invokes the procedure containing the start location $l^{\prime}$, then we prefer successor states where $o b j_{s y m}$ is initialized to objects of type $T:=\operatorname{getClass}\left(l^{\prime}\right)$. The getClass function returns the class containing the program location $l^{\prime}$. The $h_{3}\left(s_{i}^{\prime}\right):=0$ if $o b j_{\text {sym }}$ points to an object of type $T$; otherwise, $h_{3}\left(s_{i}^{\prime}\right):=1$. The information in the abstract trace allows us to intelligently pick objects of types that lead to the target location.

\section{Refinement}

The refinement process is invoked when the symbolic execution cannot reach the target of the branch statement in an abstract trace, $\pi_{i}$. The variables in the branch predicate can either be concrete, symbolic, global, or thread-local. In an effort to execute the needed branch condition in the abstract trace we add locations in the abstract system that redefine variables in the branch predicate. The algorithm for refine_trace $\left(A_{\alpha}, \pi_{i}\right)$ is shown in Fig. 5. We define some additional functions that are used to describe the refinement process.

- same_lock $\left(l_{a}, l_{b}\right)$ returns true iff acquireLock $\left(l_{a}\right) \wedge$ acquireLock $\left(l_{b}\right)$ such that $l_{a}$ and $l_{b}$ acquire the lock on the same object.

- get_abstract_trace $\left(L_{v}\right)$ returns an abstract trace from the start of a program to $l_{v} \in L_{v}$.

- $\Pi_{\alpha}$.replace_trace $\left(\pi_{i}, \pi_{j}\right)$ substitutes $\pi_{i}$ with $\pi_{j}$ in the abstract trace set $\Pi_{\alpha}$.

The refinement process is shown in Fig. 5. The first element of the abstract trace, $\pi_{i}$, is a branch statement as assumed on line 1 of Fig. 5 . To generate a set of program locations, $L_{v}$, on line 2 we select locations where thread-local and 
global variables in the branch predicate are redefined. We use an interprocedural data flow analysis to generate $L_{v}$. We compute an alias analysis through different procedures to check if variables passed to different procedures can alias the same object. We also compute the reaches definition along interprocedural paths. Note this more expensive data flow analysis is invoked on a need-to basis in the refinement phase when the original intraprocedural data flow analysis was insufficient to generate the key locations in determining the reachability of the target locations.

The fixpoint for the abstract system, $\mathcal{A}$, is recomputed to add the locations that are relevant in checking the reachability of all $l_{v} \in L_{v}$. The abstract system is now modified and contains a new set of locations and edges. The get_abstract_trace returns an abstract trace in the abstract system from the start of the program to some location in $L_{v}$. We randomly pick a $l_{v} \in L_{v}$ and generate an abstract trace from the start of the program to $l_{v}$ in $\mathcal{A}$. When there are multiple abstract traces to $l_{v}$ then we, again, randomly pick an abstract trace.

The abstract trace set $A_{\alpha}$ is updated with a new abstract trace that contains additional locations leading to the definition of a variable used in the branch predicate. In Fig. $5, \pi_{v}:=\left\langle l_{0}, \ldots, l_{v}\right\rangle$ is an abstract trace from the start of the program to location, $l_{v}$, that defines a variable in the branch predicate. The abstract trace $\pi_{\text {pre }}$ is the prefix of the trace $\pi_{i}$ in the original abstract trace set. The prefix denotes the sequence of locations from the start of the program up to, and not including, the conditional branch statement that cannot reach the desired target.

In order to generate the replacement abstract trace we check the lock dependencies between $\pi_{p r e}$ and $\pi_{v}$. If $\pi_{\text {pre }}$ and $\pi_{v}$ acquire the lock on the same object (line 6), then we add the corresponding lock relinquish location to $\pi_{v}$ (line 7). Adding the lock relinquish location ensures that if one thread acquires a lock to define a variable in the branch predicate, then after the definition another thread is not blocked trying to acquire the same lock to reach the conditional statement. A new prefix, $\pi_{n e w}$, is essentially created by combining $\pi_{v}$ and $\pi_{\text {pre }}$. This operation adds to the abstract trace the definition of a variable in the branch predicate before the conditional statement. Finally we replace in the abstract trace set $A_{\alpha}$ the abstract trace corresponding to $\pi_{\text {pre }} \circ \pi_{i}$ with $\pi_{\text {new }} \circ \pi_{i}$ (line 9 ). The guided symbolic execution is now restarted from the initial program state $s_{0}$ and guided along the updated abstract trace set.

Suppose, $A_{\alpha}:=\left\{\left\langle l_{0}, l_{1}, l_{2}, l_{3}, l_{4}, l_{5}\right\rangle\right\}$ and $\pi_{i}:=\left\langle l_{4}, l_{5}\right\rangle$ for the example in Fig. 2. In the runtime environment we have found a feasible execution trace that visits locations $l_{0}$ to $l_{3}$, but at the conditional branch $l_{4}$ the execution cannot reach the desired target location $l_{5}$. The refinement process shown in Fig. 5 adds new locations and edges shown in Fig. 3(b) to the abstract system in addition to the ones shown in Fig. 3(a). In Fig. 3(b) location $\alpha_{5}$ defines the integer field, $e$, of the shared variable elem; $\pi_{v}:=\left\langle\alpha_{0}, \alpha_{1}, \alpha_{2}, \alpha_{3}, \alpha_{4}, \alpha_{5}\right\rangle$ such that the sequence leads from the start of the program to $\alpha_{5}$ in Thread B. The prefix of $\pi_{i}$ is $\pi_{\text {pre }}:=\left\langle l_{0}, l_{1}, l_{2}, l_{3}\right\rangle$. Locations $l_{1}$ and $\alpha_{2}$ in Fig. 3(a) and Fig. 3(b) respectively 


\begin{tabular}{|l|l|r|r|r|r|}
\hline & Error Type & SLOC & $\begin{array}{r}\text { Time } \\
(\text { Tracs })\end{array}$ & $\begin{array}{r}\text { Trace } \\
\text { Sets }\end{array}$ & $\begin{array}{r}\text { Memory } \\
\text { MB }\end{array}$ \\
\hline Reorder & Reachability & 44 & 0.28 & 5 & $1.93 \mathrm{MB}$ \\
\hline Airline & Reachability & 31 & 0.30 & 3 & $1.58 \mathrm{MB}$ \\
\hline VecDeadlock0 & Deadlock & 7267 & 1.21 & 5 & $38 \mathrm{MB}$ \\
\hline VecDeadlock1 & Deadlock & 7169 & 0.98 & 17 & $38 \mathrm{MB}$ \\
\hline VecRace & Race & 7151 & 0.92 & 8 & $39 \mathrm{MB}$ \\
\hline
\end{tabular}

Table 1. Information on models and abstract trace generation.

acquire the lock on the same object elem; hence, we add the lock release location to $\pi_{v}:=\pi_{v} \circ \alpha_{6}$. Finally the guided symbolic execution is restarted from $s_{0}$ and $A_{\alpha}:=\left\{\pi_{v} \circ \pi_{\text {pre }} \circ \pi_{i}\right\}$.

The refinement process can be invoked repeatedly for the same branch condition it is possible the same definition of the variable is added multiple times. Such a scenario allows us to handle the cases where the variable needs to be over a certain value in the branch predicate and its value is incremented by some variable or constant in the definition. The refinement strategy is in itself a heuristic. Developing and evaluating other refinement strategies is a work in progress.

\section{$7 \quad$ Experimental Results}

We conduct experiments on machines with $8 \mathrm{~GB}$ of RAM and two Dual-core Intel Xeon EM64T processors $(2.6 \mathrm{GHz})$. We use the symbolic extension of the Java PathFinder (JPF) v4.1 model checker with partial order reduction turned on [16]. JPF uses a modified JVM that operates on Java bytecode. The abstractionrefinement and the heuristic computation processes are also performed on the Java bytecode. This allows us to model any libraries used by the program as part of the multi-threaded system.

We present an empirical study on five multi-threaded Java programs shown in Table 1. The Reorder and the Airline model are benchmarked examples while the VecDeadlock0, VecDeadlock1, and VecRace are examples that use the JDK 1.4 synchronized Vector library in accordance with the documentation. The programs are part of the JPF examples repository and can be obtained by downloading JPF from [10]. We use Jlint to automatically generate warnings on possible deadlocks and race-conditions in the synchronized Vector library [2]. The Reorder and Airline programs have user-defined reachability properties. The errors in the Vector library are caused by a combination of data values and thread schedules. For each program, in Table 1, we show the type of error, source lines of code (SLOC), total time taken in seconds to generate the set of abstract traces (Time), total number of abstract trace sets tested (Traces Sets), and total memory used (Memory). The parameters with the program names indicate the thread configuration of a particular program. Each parameter represents the total number of symmetric threads in the system. 


\begin{tabular}{|l|r|r|r||r|r|}
\hline Model & States & $\begin{array}{r}\text { Time } \\
\text { secs }\end{array}$ & $\begin{array}{r}\text { Memory } \\
\text { MB }\end{array}$ & $\begin{array}{r}\text { Total trace } \\
\text { Length }\end{array}$ & $\begin{array}{r}\text { Total } \\
\text { Refinements }\end{array}$ \\
\hline Reorder $(9,1)$ & 205 & 0.874 & $7 \mathrm{MB}$ & 13 & 1 \\
\hline Reorder $(10,1)$ & 239 & 0.875 & $7 \mathrm{MB}$ & 13 & 1 \\
\hline Airline $(15,3)$ & 619 & 0.548 & $5 \mathrm{MB}$ & 3 & 13 \\
\hline Airline $(20,2)$ & 1230 & 0.521 & $6 \mathrm{MB}$ & 3 & 19 \\
\hline Airline $(20,1)$ & 1248 & 0.498 & $6 \mathrm{MB}$ & 3 & 20 \\
\hline VecDealock0 & 37 & 1.003 & $66 \mathrm{MB}$ & 14 & 1 \\
\hline VecDeadlock1 & 294 & 1.005 & $69 \mathrm{MB}$ & 15 & 2 \\
\hline VecRace & 312 & 1.032 & $65 \mathrm{MB}$ & 12 & 1 \\
\hline
\end{tabular}

Table 2. Effort in error discovery and abstract trace statistics.

Picking Abstract Traces: A large number of abstract trace sets can be generated from the abstract system. To pick the initial abstract trace sets we choose sets that contain traces with the smallest number of call sites leading from the start of the program to each target location. Several abstract trace sets may contain the smallest number of call sites. In Table 1, the number of abstract trace sets reported are the ones generated for the smallest number of call sites. For the programs used in this empirical study, we were able to discover the goal state by simply using the initial abstract trace sets.

We present in Table 2 the results for generating a concrete feasible execution trace corresponding to an abstract trace for the programs used in our empirical study. The guided symbolic execution trials for the different abstract trace sets reported in Table 1 are launched in parallel on different computation nodes since each trial is completely independent of the other trials. When a feasible execution trace is generated along an abstract trace set, we terminate the other trials. We present the total number of states generated, total time taken, and total memory used in the trial that generates a feasible execution trace corresponding to the abstract trace set. We also show the length of the initial trace $\left(\sum_{\pi_{i} \in \Pi_{\alpha}}\left|\pi_{i}\right|\right)$ and total number of refinements performed on the abstract trace; $\Pi_{\alpha}$ is the input abstract trace set.

The results in Table 2 indicate that the guided symbolic execution technique can quickly generate a concrete feasible execution to a corresponding abstract trace. In the VecDeadlock0, the technique only generates 37 states and takes about 1 second to find the deadlock in the program. Similarly in the VecDeadlock1 and VecRace programs, the guided symbolic execution only generates a few hundred states before generating a concrete trace to the error. Exhaustive symbolic execution using a depth-first search is unable to discover the errors in the programs used in the empirical study within a time bound of one hour.

In the examples shown in Table 2 most models require only one or two refinements to find the goal state. In these models one or two perturbations to global variables were required to elicit the errors in the system. A related heuristic, the iterative context-bounding approach bounds the number of preemptions along a certain path in order to reach the error faster [12]; however, for the configurations of the models used in this study the iterative context-bounding approach with a 
preemption bound of two, the Chess concurrency testing tool was unable to find the error in a time-bound of one hour even with no data non-determinism [13]. Note that we created corresponding $C \#$ programs. The Airline model required a larger number of refinements because the reachability of the target location depends on the value of a global counter that is modified by different threads. In this case even with the correct number of preemptions as the input the iterative context-bounding approach was unable to discover an error. ConTest, [6], was also unable to find an error in the models with the thread configurations used in the empirical study in a 1000 trails (these experiments too were conducted in the absence of data non-determinism).

\section{Related Work}

Recent work by Tomb et al. uses symbolic execution to generate concrete paths to null pointer exceptions at an inter-procedural level in sequential programs [24]. In contrast, concolic testing executes the program with random concrete values in conjunction with symbolic execution to collect the path constraints over input data values $[23,22]$. The cost of constraint solving in concolic testing to achieve full path coverage in a concurrent system is extremely high. The techniques presented in this work are complementary to concolic testing. The techniques can also be used to efficiently guide concolic testing.

Recent work shows that guiding the concrete program execution along a sequence of manually generated program locations relevant in verifying the feasibility of the target location dramatically lowers the time taken to reach the target location [18]. The manual aspect of generating relevant program locations is tedious and sometimes intractable.

Race-directed random testing of concurrent programs uses the output of imprecise dynamic analysis tools and randomly drives threads to the input locations [21]. The work in [18] shows that guiding the search through key locations relevant in determining the target locations yields significantly better error discovery rates. Dynamic analysis tools such as ConTest use heuristics to randomly add perturbations in the thread schedules [6]. The results are similar to those obtained with just a stateless random search and it is not very effective in error discovery. Chess is a concurrency testing tool that systematically explores thread schedules in $C \#$ programs and supports iterative context bounding [12].

Model checking is a formal approach for systematically exploring all possible behaviors of a concurrent software system $[9,17,25,3]$. The state space explosion problem renders it intractable in verifying medium to large-sized programs. Conservative abstractions are applied to high-level programming languages $[8,3]$ in order to verify programs. The abstraction is iteratively refined if it generates an infeasible counter-example to an error state. Counter-example guided abstraction refinement techniques are successful in verifying sequential programs; however, they are not effective for testing concurrent programs.

Related works in hardware verification guide the simulation of the concrete model using an abstract model of boolean variables that represent the transition 
relation [14,15]; however, these works are limited to verifying circuit designs and boolean programs. The techniques cannot be extended to verify complex concurrent software systems. Another area of related work is the use of abstract databases and heuristics that are used to guide the searches in planning problems [4].

There is a large body of related work aimed at verifying and testing concurrent programs. A detailed discussion on various related works is provided in a technical report [20].

\section{Conclusions and Future Work}

In this work we present an abstraction-guided symbolic execution technique that efficiently detects errors caused by thread schedules and data values in concurrent programs. Based on a set of input target locations the technique automatically generates an abstract system that contains relevant locations in checking the reachability of the target locations. The symbolic execution is guided along traces in the abstract system to generate a corresponding feasible execution path to the target locations. Heuristics are used to efficiently rank thread and data non-determinism to guide the symbolic execution along the locations in the abstract system. We empirically show that the abstraction-guided refinement technique can find errors in multi-threaded Java programs in a few seconds where exhaustive search techniques are unable to find errors within a time bound of an hour.

In the case when we are unable to discover a feasible execution path, we want to design a probabilistic measure to estimate the likelihood of the reachability of the target locations as future work. Another avenue of future work consists of studying more precise refinement techniques based on compositional symbolic execution [1].

\section{References}

1. S. Anand, P. Godefroid, and N. Tillmann. Demand-driven compositional symbolic execution. In C. R. Ramakrishnan and J. Rehof, editors, TACAS, volume 4963 of LNCS, pages 367-381. Springer, 2008.

2. C. Artho and A. Biere. Applying static analysis to large-scale, multi-threaded java programs. In Proc. $A S W E C$, page 68, Washington, DC, USA, 2001. IEEE Computer Society.

3. T. Ball and S. Rajamani. The SLAM toolkit. In G. Berry, H. Comon, and A. Finkel, editors, Proc. CAV, volume 2102 of LNCS, pages 260-264, Paris, France, July 2001. Springer-Verlag.

4. S. Edelkamp. Planning with pattern databases. In Proc. European Conference on Planning, pages 13-24, 2001.

5. D. Engler and K. Ashcraft. RacerX: effective, static detection of race conditions and deadlocks. In Proc. SOSP '03, pages 237-252, New York, NY, USA, 2003. ACM Press. 
6. Y. Eytani, K. Havelund, S. D. Stoller, and S. Ur. Towards a framework and a benchmark for testing tools for multi-threaded programs: Research articles. Concurr. Comput. : Pract. Exper., 19(3):267-279, 2007.

7. P. Godefroid. Model checking for programming languages using verisoft. In Proc. of POPL, pages 174-186, New York, NY, USA, 1997. ACM.

8. T. A. Henzinger, R. Jhala, R. Majumdar, and G. Sutre. Software verification with Blast. In T. Ball and S. Rajamani, editors, Proc. SPIN Workshop, volume 2648 of LNCS, pages 235-239, Portland, OR, May 2003.

9. G. J. Holzmann. The SPIN Model Checker: Primer and Reference Manual. Addison-Wesley, 2003.

10. Java PathFinder Website. http://javapathfinder.sourceforge.net.

11. J. C. King. Symbolic execution and program testing. Communications of the ACM, 19(7):385-394, 1976.

12. M. Musuvathi and S. Qadeer. Iterative context bounding for systematic testing of multithreaded programs. SIGPLAN Not., 42(6):446-455, 2007.

13. M. Musuvathi and S. Qadeer. Fair stateless model checking. In Proc. of PLDI, pages 362-371, New York, NY, USA, 2008. ACM.

14. K. Nanshi and F. Somenzi. Guiding simulation with increasingly refined abstract traces. In Proc. DAC, pages 737-742, New York, NY, USA, 2006. ACM.

15. F. M. D. Paula and A. J. Hu. An effective guidance strategy for abstraction-guided simulation. In Proc. DAC '07, pages 63-68, New York, NY, USA, 2007. ACM.

16. C. S. Păsăreanu, P. C. Mehlitz, D. H. Bushnell, K. Gundy-Burlet, M. Lowry, S. Person, and M. Pape. Combining unit-level symbolic execution and systemlevel concrete execution for testing NASA software. In Proc. ISSTA, pages 15-26, New York, NY, USA, 2008. ACM.

17. Robby, M. B. Dwyer, and J. Hatcliff. Bogor: An extensible and highlymodular model checking framework. ACM SIGSOFT Software Engineering Notes, 28(5):267-276, September 2003.

18. N. Rungta and E. G. Mercer. A meta heuristic for effectively detecting concurrency errors. In Haifa Verification Conference, Haifa, Israel, 2008.

19. N. Rungta and E. G. Mercer. Guided model checking for programs with polymorphism. In Proc. PEPM, pages 21-30, New York, NY, USA, 2009. ACM.

20. N. Rungta, E. G. Mercer, and W. Visser. Efficient testing of concurrent programs with abstraction-guided symbolic execution, 2009. Technical Report, available online at http://vv.cs.byu.edu/publications/papers/abstraction.pdf.

21. K. Sen. Race directed random testing of concurrent programs. SIGPLAN Not., 43(6):11-21, 2008.

22. K. Sen and G. Agha. A race-detection and flipping algorithm for automated testing of multi-threaded programs. In Proc. HVC, volume 4383 of LNCS, pages 166-182. Springer, 2007.

23. K. Sen, D. Marinov, and G. Agha. CUTE: a concolic unit testing engine for C. In Proc. ESEC/FSE-13, pages 263-272, New York, NY, USA, 2005. ACM.

24. A. Tomb, G. Brat, and W. Visser. Variably interprocedural program analysis for runtime error detection. In Proc. ISSTA, pages 97-107, New York, NY, USA, 2007. ACM Press.

25. W. Visser, K. Havelund, G. P. Brat, S. Park, and F. Lerda. Model checking programs. Automated Software Engineering, 10(2):203-232, 2003. 